\title{
Ad-Hoc Business Processes in Web Services
}

\author{
Andreas Wombacher Bendick Mahleko \\ Fraunhofer Institute \\ Integrated Publication and Information Systems (IPSI) \\ D-64293 Darmstadt, Germany \\ http://ipsi.fhg.de/oasys/ \\ \{wombach|mahleko\}@ipsi.fhg.de
}

\begin{abstract}
Web service technologies promise to facilitate collaboration among business partners by helping potential business partners find one another and integrate their business processes to do business. Daunting challenges however exist before the full potential of Web services can be realized. Indexing mechanisms for complex workflows are needed for example to be able to efficiently search and match business partners on the basis of compatible workflows. Business collaborations invariably comprise multiple-parties, and the process of aggregating workflows belonging to different parties to establish a global agreement in an ad-hoc manner presents another challenge. We also need to consider the decision problem how to determine, during the setting-up of a global agreement, whether some services must be fulfilled by the same provider or not. In this paper we highlight these challenges and propose possible solutions.
\end{abstract}

\section{Introduction}

Web services promise to provide an infrastructure that will be a basis on which organizations will find one another and collaborate to do business. This is made possible by the use of open Web-based standards like SOAP [4], WSDL [5], UDDI [1] and BPEL [6]. SOAP is a standard based on XML and is used for exchanging messages within the web service infrastructure. WSDL is an XML-based description for services and UDDI is a standard for publishing and discovering services on the Web. BPEL represents a convergence of XLANG [12] and WSFL [10] specifications and it is used to specify business processes as services. Using BPEL organizations can describe internal and external parts of their workflows so that they can be recognized as web services. The public parts of the workflows are used for collaboration with external business partners while the private parts are used for modeling those parts of the business process the organization does not want to expose to potential competitors. Although much work has been done, a number of challenges still remain before web services can be used to their full potential. There exists challenges related to annotating service descriptions and their usage within searching and matchmaking of services fitting particular business processes. A further challenge deals with dynamically establishing trading agreements within multi-lateral collaborations. Currently, no model exists to analyze or simulate the process of agreement formation involving multiple parties. Another challenge concerns the unification of service instances that is the determination if certain services must be provided by the same instance or not. Solving this unification decision problem using limited workflow specification information only is a non-trivial task.

The rest of the paper is organized as follows: Section 2 presents the description of the problems to be solved, Section 3 presents the approach for solving the identified problems and Section 4 is about related work. Section 5 gives the conclusion and an insight on future work.

\section{Problem Description}

In this section we elaborate on the challenges we briefly mentioned in the introductory section. These are:

- the need for searching and matchmaking engines that support service descriptions with workflow specific annotations,

- the need for concepts dealing with establishing meaningful and consistent multi-lateral collaborations based on bilateral agreements,

- the need to develop tools for deciding unification of service instances in multi-lateral collaborations.

In the following sections, we will examine each of these issues in more detail. 


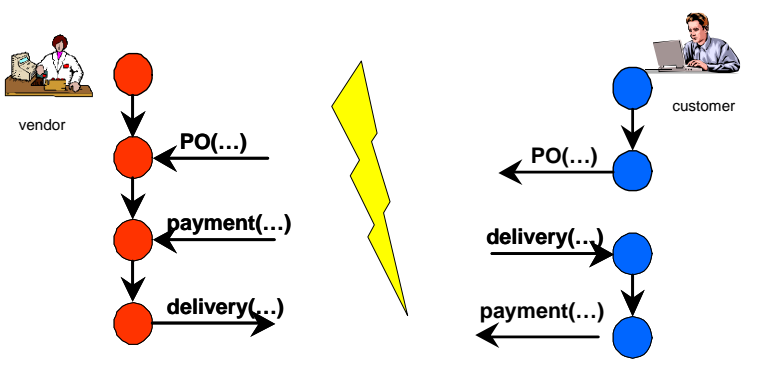

Figure 1. Vendor and Customer Workflows

\subsection{Searching and Matchmaking of Business Pro- cesses}

The example in Figure 1 shows two parties that want to collaborate to do business. One represents a vendor organization and the other a customer organization. Each of the organizations has a local workflow that it uses to carry out its business operations, i.e., internal procedures for doing business. In addition, each organization has an external part of its workflow that it uses to collaborate with external business partners, e.g., to procure goods. The figure does not show details of the local workflows, but instead shows only the external parts used for interaction (collaboration protocol). The external part of the vendor workflow works as follows: first, a purchase order request is received from a customer. Next, the vendor system awaits a payment message from the organization that sent a purchase order. Finally, the goods are delivered to the buying organization, and a delivery message is send. On the customer organization side, the following workflow exists: first, a customer sends a purchase order request to the vendor organization; next, the customer organization expects to receive a delivery message indicating that the goods are being delivered, along with the delivery details. Finally, the customer organization pays for the purchased goods.

It is obvious that the two external parts of the workflows do not match, also they match on behalf of the WSDL specification because of the usage of the same messages. Thus, the service description published in the repository must be extended by collaboration protocol annotations specified e.g., in conversation languages like WSCL [3]. We are convinced, that an automatic generation of conversation specifications based on a local workflow specifications increases user acceptance.

\subsection{Establishing Contracts in Ad-Hoc Multi- Lateral Collaborations}

Figure 2 shows a procurement example involving the following trading parties: customer, vendor, shipping com- pany and bank. The aim of the customer is to buy some goods from a vendor using his credit card and potentially to do an online tracking of the shipped goods. The vendor intends to sell goods to a customer and to use an external shipping service. The two remaining parties (bank and shipper) offer core services, that is different payment methods and shipping goods including a parcel tracking.

Like in the previous example, each of the parties has his own local workflow and provides respectively request services from other trading parties, without knowing their local workflows. The main challenge is to set-up an ad-hoc multi-lateral collaboration based on several local workflows resulting in a consistent and meaningful global workflow. From Figure 2, the global workflow can be understood and executed quite easily, but the global workflow does not exist a priori. None of the parties involved provides or even knows for sure the global workflow. Further, not all of the parties are willing to provide their local workflows to trading partners, because mission critical information may be contained. Thus, the global workflow cannot be composed from the local ones, but must be determined based on limited workflow information (collaboration protocols) provided by each party.

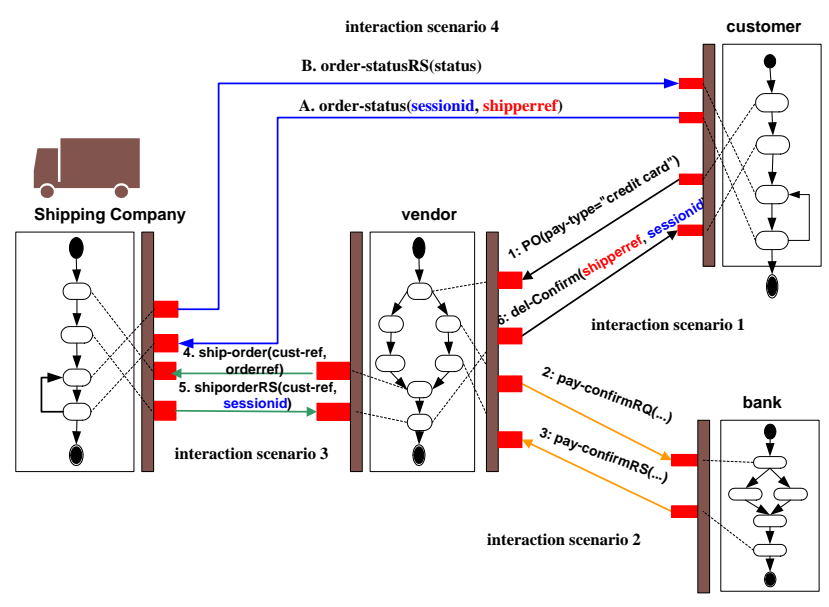

Figure 2. Multi-Lateral Collaboration

In particular, no optimal plan for setting up a global multi-lateral contract can be calculated, because no global workflow is available and the plan calculation is based on partial information only, which is definitely not sufficient. The way of influencing the contracting is limited to the selection of trading partners, which affects the number of potential ways to set-up a contract, which also affects the number of potential different kinds of comparisons of collaboration protocols. This calls for concepts modeling the establishment of a contract involving interested parties, which 
are currently not considered in the web service infrastructure, dealing with searching and matchmaking of services involved in multi-lateral collaborations, and modeling of contracts and service level agreements themselves as well as determining of a concept how to set them up to a multilateral contract.

\subsection{Unification of Service Instances}

We pick-up just a single issue related to searching and matchmaking within multi-lateral collaborations which are based on a non-existing global workflow model: unification of service instances in service discovery. Lets assume two parties (a vendor and a customer) agreed on a partial contract each of them requesting a further service. Service discovery now must determine, whether the two requested services must be provided by a single service provider instance or can be provided by two independent service provider instances.

Figure 3(a) shows an example where each of the parties requires a shipper service: one for interacting with a vendor to make delivery requests and the other for interacting with a customer to do parcel tracking. From the semantic understanding of the scenario and the implicitly obvious global workflow (as depicted in Figure 2), we know that the two services must be fulfilled by the same shipping provider service instance, because the services rely on a common state (as depicted in Figure 3(b) ). Solving this decision problem based on collaboration protocol specifications only without having a global workflow is a major challenge.

\section{Approach}

This section presents our current results addressing some of the problems identified in previous sections. Unfortunately, we can not provide complete solutions, but describe the ongoing work on partial aspects of the challenges.

\subsection{Searching and Matchmaking of Business Pro- cesses}

The matchmaking problem itself can be understood as a subsumption problem of deterministic finite state automatons (DFAs), that is checking whether the messages supported by a service provider at least contains the subset of message sequences understood by the service user, which is based on standard DFA algorithms for subsumption checking as described in [9].

In [14] we present an approach to automatically derive the collaboration protocol specification based on a local workflow model and use this within matchmaking. Because we based the approach on statecharts, it can quite easily be adapted to different workflow modeling languages like

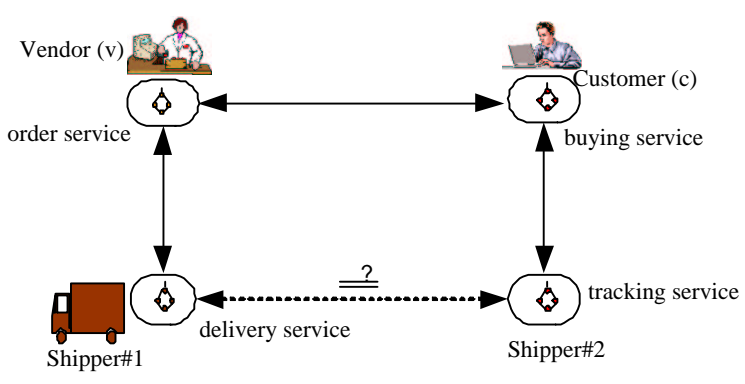

(a) Delivery and Tracking Services. Are The 2 Services Fufilled by the Same Provider?

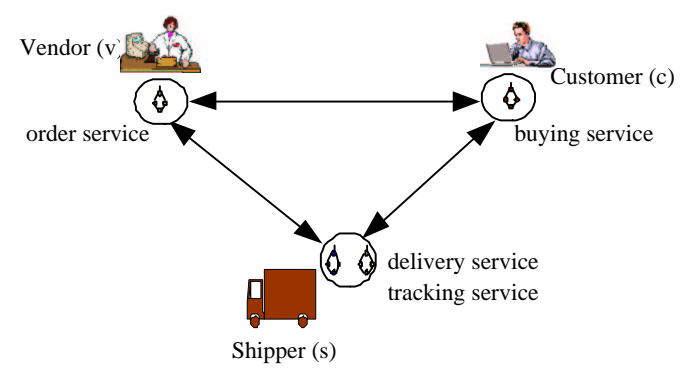

(b) Delivery and Tracking Services with the 2 Services Fufilled by the Same Provider.

\section{Figure 3. Checking for Provider Equivalence}

BPEL [6], WSFL [10] or XLANG [12]. Figure 4 illustrates our approach: First a service provider expresses his local workflow using BPEL, WSFL or XLANG and, a view representation of the public part of the workflow can be calculated from the private workflow. A view of a role represents the public part of a workflow that is needed for interaction with that role. The service provider stores this view in a public repository like UDDI using standards like WSCL to describe the supported message patterns.

If another partner (called the service requestor) is searching for a suitable business partner, i.e., a business partner that supports his message sequences, he also calculates the view of the partner he wants to interact with to determine the message sequences to be exchanged. In addition, he also calculates the perspective of the provider partner. The perspective of the provider represents a minimum workflow needed to interact with the service provider. From this minimum workflow of the service provider, we now need to find service providers who can subsume it. The criteria whether or not a matching service provider can be found is therefore determined by the fact that a service provider whose workflow subsumes the workflow generated by the service requestor can be found or not. 


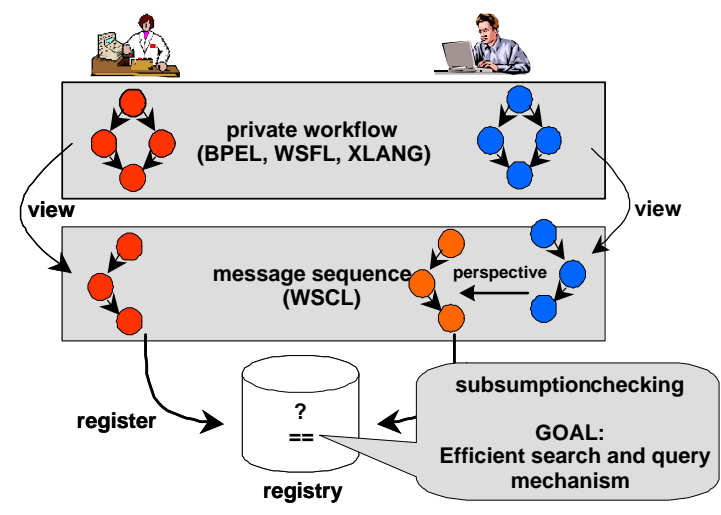

Figure 4. Workflow-Based Service Discovery

\subsection{Establishing Contracts in Ad-Hoc Multi- Lateral Collaborations}

Establishing multi-lateral ad-hoc collaborations based on local workflows is a highly combinatorial problem, which has quite a lot of different facets each representing serious problems. Exemplary we name the contract establishment process under consideration of service level agreements, the calculation of a plan for setting-up a collaboration, and the definition of searching and matchmaking strategies during the process of setting-up a multi-lateral collaboration.

To get a better understanding of the problem domain of multi-lateral collaborations, we investigated dependencies within these collaborations and came-up with a classification scheme, which will be published in [15]. We found two types of dependencies: service dependencies influencing the global workflow and role dependencies influencing the need of service instance unification (see next subsection).

Within the dimension of service dependencies, we found three cases:

\subsubsection{Fully Centralized Model}

The full centralized model requires a single party acting as an intermediary by combining and coordinating further non-interrelated service providers. The intermediary has the total control for setting-up the collaboration, because no interdependencies between further services exist.

\subsubsection{Decentralized Model}

The decentralized model does not restrict the interrelations between the different service providers and therefore provides complete openness and full combinatoric number of options during the contract establishment process.

\subsubsection{Partially Centralized Model}

The partially centralized model is a mixture out of the above two. An exemplary scenario is described in Figure 2 explaining a simple procurement scenario involving a parcel tracking.

Based on this classification, we continue our work in searching and matchmaking as well as contract establishment by defining matchmaking functions and investigating local influences on the contract establishment process by exemplary dynamically adding constraints to the contracting process.

\subsection{Unification of Service Instances}

The issue of role dependencies influences the need of service unification. Unification itself is a well known problem (e.g., [2]), which itself has a high computational complexity. Things getting worse, when applying unification of services to collaboration protocols (DFAs). The intuitive approach separates each potential message sequence and apply the unification algorithm to it. This approach becomes infinite in case the message sequences contain cycles.

Our approach currently relies on the use of structural information of the involved workflows to come up with a single merged workflow that exhibits the behavior of both workflows. We achieve this by carrying out a series of transformations on the original workflows, and at the same time making sure that structural information that describes the behavior of the provider is not lost. Then, we check the resulting graph on dependencies and use this as a necessary condition for unification. The satisfying condition is still missing, but we continue working on it.

\section{Related Work}

Definitely, there exists related work, which we at least partly investigated for other publications like [14] and [15].

In addition, on the issue of plan generation and checking if two services are offered by the same provider or not, existing B2B e-commerce applications do not deal with this type of problem, because they rely on personally negotiated frame contracts based on global workflow models provided by de-facto standardization organizations like RosettaNet [11], ebXML [8] or cXML [7]. In addition to industrial de-facto standardization bodies, also most interorganizational workflow research approaches are based on global workflow definitions, like [13]. These approaches are not facing the problem addressed within this paper because of the global workflow model which is not available in multilateral ad-hoc collaborations. 


\section{Conclusion}

In this paper, we highlighted the challenges that still inhibit the use of web services to support ad-hoc business processes and how these challenges might be overcome. We are currently focusing on indexing mechanisms for collaboration protocols derived from complex workflows for efficient searching and querying of complex services. Further, we are addressing the issue of establishing multi-lateral adhoc collaborations and a few of the related issues.

\section{References}

[1] I. Ariba, I. Corporation, and M. Corporation. Universal description, discovery and integration, September 2000. http://www.uddi.org/.

[2] F. Baader and J. Siekmann. Unification theory. In D. M. Gabbay, C. J. Hogger, and J. A. Robinson, editors, Handbook of Logic in Artificial Intelligence and Logic Programming, volume 2, Deduction Methodologies, pages 41-125. Oxford University Press, Oxford, UK, 1994.

[3] A. Banerji, C. Bartolini, D. Beringer, V. Chopella, K. Govindarajan, A. Karp, H. Kuno, M. Lemon, G. Pogossiants, S. Sharma, and S. Williams. Web services conversation language (wscl) 1.0 w3c note, March 2002. http://www.w3.org/TR/wscl10/.

[4] D. Box, D. Ehnebuske, G. Kakivaya, A. Layman, N. Mendelsohn, H. F. Nielsen, S. Thatte, and D. Winer. Simple object access protocol (soap) $1.1 \mathrm{w} 3 \mathrm{c}$ note, May 2000. http://www.w3.org/TR/SOAP/.

[5] E. Christensen, F. Curbera, G. Meredith, and S. Weerawarana. Web services description language (wsdl) $1.1 \mathrm{w} 3 \mathrm{c}$ note, March 2001. http://www.w3.org/TR/wsdl.

[6] F. Curbera, Y. Goland, J. Klein, F. Leymann, D. Roller, S. Thatte, and S. Weerawarana. Business process execution language for web services, version 1.0, July 2002. ftp://www6.software.ibm.com/software/developer/library/wsbpel.pdf.

[7] cXML. Commerce xml resources. http://www.cxml.org/.

[8] ebXML. ebxml standardization. http://www.ebxml.org/.

[9] J. E. Hopcroft, R. Motwani, and J. D. Ullman. Introduction to Automata Theory, Languages, and Computation. Addison Wesley, 2001.

[10] F. Leymann. Web services flow language (wsfl 1.0), May 2001. http://www3.ibm.com/software/solutions/webservices/pdf/WSFL.pdf.

[11] RosettaNet. Rosettanet homepage. http://www.rosettanet.org/.

[12] S. Thatte. Xlang web services for business process design. http://www.gotdotnet.com/.

[13] W. van der Aalst and M. Weske. The P2P approach to interorganizational workflows. In Proc. of 13. Int. Conf. on Advanced Information Systems Engeneering (CAISE'01), Interlaken, Switzerland, 2001.

[14] A. Wombacher and B. Mahleko. Finding trading partners to establish ad-hoc business processes, 2002.

[15] A. Wombacher and B. Mahleko. Classification of ad-hoc multi-lateral collaborations based on local workflow models. In ACM SAC 2003 proceedings, 2003. To Appear. 